\title{
Shielding Design for the South Pole nToF Diagnostic at the NIF
}

\author{
Hesham Khater ${ }^{*}$, Shiva Sitaraman, James Hall, Robert Hatarik, Joseph Caggiano, and Cory Waltz \\ Lawrence Livermore National Laboratory, Livermore, CA 94550, USA
}

\begin{abstract}
Neutron time of flight (nToF) detectors are fielded at the National Ignition Facility (NIF) to measure neutron yield, ion temperature, and downscattering in the cold fuel for D-T implosions. Anisotropically assembled cold fuel may generate different $\mathrm{nToF}$ data when measured by detectors located at the Target Chamber equator and poles. A collimated nToF line of sight has been fielded near the Target Chamber South Pole (SP) to examine any possible anisotropy in the cold fuel. The SP nToF detector is located in the lowest floor level of the NIF's Target Bay and at a distance of $\sim 18 \mathrm{~m}$ from the Target Chamber Center. The detector utilizes a solid bibenzyl scintillator and four photomultiplier tubes. The line of sight includes a port collimator that is attached to the Target Chamber and a bore hole collimator in the concrete floor above the detector. In addition, a beam line get lost hole is constructed in the Target Bay floor to minimize the backscattered radiation at the detector location. Initial measurements indicated the need for installation of additional shielding to eliminate gamma background during the period before arrival of the 14.1 MeV neutrons to the detector. A set of MCNP Monte Carlo simulations with the full Target Bay model were conducted to provide an estimate of the expected neutron and gamma backgrounds during D-T shots. A new shielding scheme is designed to reduce the gamma background by an order of magnitude.
\end{abstract}

\section{Introduction}

The National Ignition Facility (NIF) at Lawrence Livermore National Laboratory is the world's largest and most powerful laser system for inertial confinement fusion. The NIF is a 192 laser beam facility capable of producing $1.8 \mathrm{MJ}, 500 \mathrm{TW}$ of ultraviolet light. The NIF is configured for "indirect drive" approach where highenergy laser beams are focused to enter a small metal can (hohlraum) held at the center of the Target Chamber (TC). The laser beams heat the interior surface of the hohlraum to create intense flux of thermal x-rays that ablates the surface of a fusion capsule located at the center of the hohlraum. The capsule interior contains a solid cryogenic Deuterium-Tritium (D-T) fuel layer surrounding a central low-density D-T gas. Ablation of the exterior layer of the capsule compresses the remaining capsule and the fuel. In addition to the 14.1 $\mathrm{MeV}$ neutrons produced by the D-T fusion, inelastic collisions of these neutrons with the compressed fuel and/or ablator results in "down-scattered" (DS) neutrons in the 10-12 MeV spectral range.

A smaller fraction of "reaction-in-flight" (RIF) neutrons (with energy up to $\sim 30 \mathrm{MeV}$ ) are produced by up-scattered deuterons or tritons undergoing D-T reaction with thermal ions. The full spectrum of neutrons can be measured using neutron time of flight (nToF) detectors. Anisotropically assembled cold fuel may generate different neutron time of flight data when measured by detectors located at the TC equator and poles. Several nToF detectors at different lines of sight are fielded on the NIF to measure the neutron spectrum with high accuracy [1-3]. The nToF diagnostics measure the neutron yield as a function of neutron energy by recording the detector signal strength as a function of time. For a specific distance from the source, a neutron arrival to the detector correlates to the neutron energy. The nToF diagnostics utilize scintillation detectors coupled to photomultiplier tube (PMT). The scintillators used at the NIF are characterized with low afterglow and are surrounded by light materials to reduce background from neutron scattering. Reducing scattering from nearby masses is important for accurate measurements of the DS neutrons. The detector signal is recorded by using a high bandwidth multi-channel digital oscilloscope. The $\mathrm{nToF}$ detectors are used to obtain spectral information such as ion temperature and the ratio of DS neutrons relative to the primary neutron yield.

There are seven main floor levels inside the NIF Target Bay (TB). The main floors are located at elevations of $-10.29 \mathrm{~m}$ (-33'9"), -6.63 m (-21'9"), -1.07 m (-3'6”), 5.33 m (17'6"), 8.99 m (29'6”), $12.19 \mathrm{~m}$ (40'0"), and $15.39 \mathrm{~m}\left(50{ }^{\prime} 6 "\right)$ with respect to the ground level. The TC is located in the middle of the TB with its center at an elevation of a $7.01 \mathrm{~m}$ (23'0") from the ground level. A collimated nToF line of sight (LOS) has been fielded near the TC South Pole (SP) using the TC port $\left(\theta=161^{\circ}, \phi=56.25^{\circ}\right)$. The goal of the LOS is to examine any possible anisotropy in the cold fuel. The SP $\mathrm{nToF}$ detector is located in the lowest floor level of the TB and at a distance of $\sim 18 \mathrm{~m}$ from the Target Chamber Center (TCC). The detector utilizes a solid bibenzyl

\footnotetext{
Corresponding author: khater1@1lnl.gov
} 
scintillator and four photomultipliers (PMT). Figure 1 shows the SP nToF system layout.

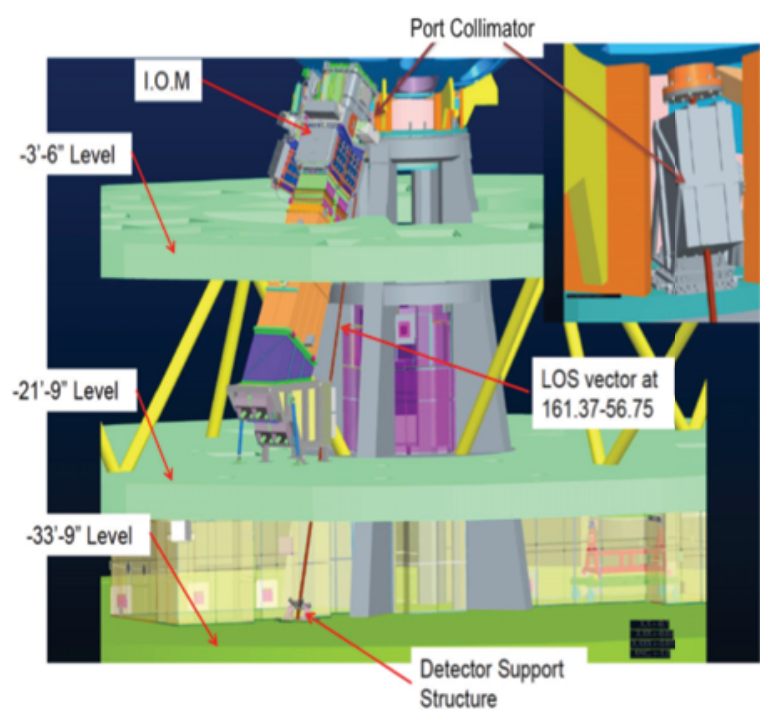

Figure 1. South Pole nToF system layout.

The LOS is collimated through the use of apertures to minimize contributions from neutrons and gammas scattered off the target positioner and diagnostic instrument manipulator (DIM) instruments from reaching the detector. The target positioner and the DIM instruments are inserted inside the TC at a few centimeters from the TCC. Contribution from radiation scattered off the TC wall is collimated through the use of a port collimator that is attached to the TC port. A twodimensional view of the port collimator's MCNP model is shown in Fig. 2.

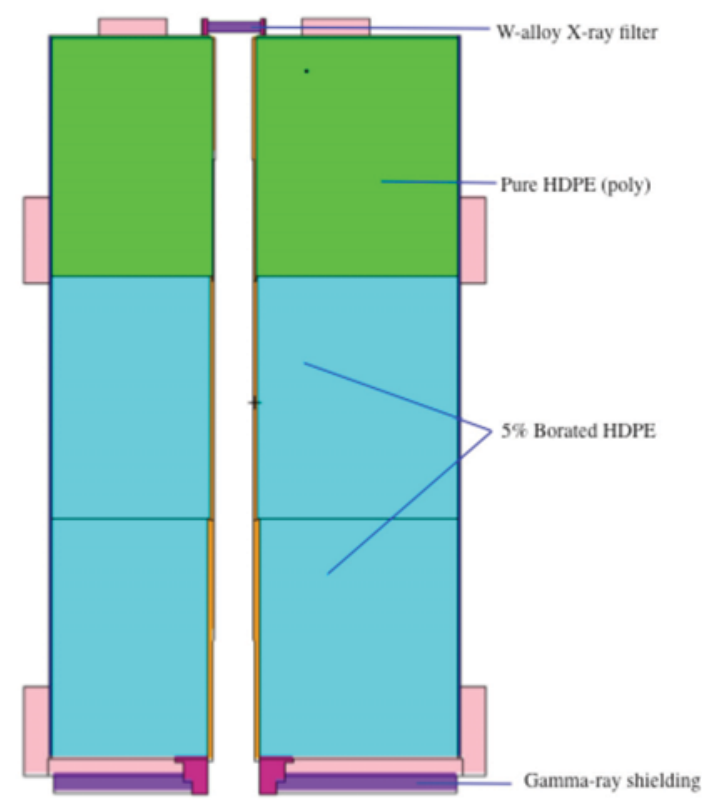

Figure 2. MCNP model of the TC port collimator.

Additional collimation is achieved through the installation of another collimator inside the $11.43 \mathrm{~cm}$ diameter bore hole in the -21'9" concrete floor above the detector. Finally, as shown in Fig. 3, a beam line get lost is constructed in the TB $-33^{\prime}$ '9" floor to minimize/delay backscattered radiation at the detector location.

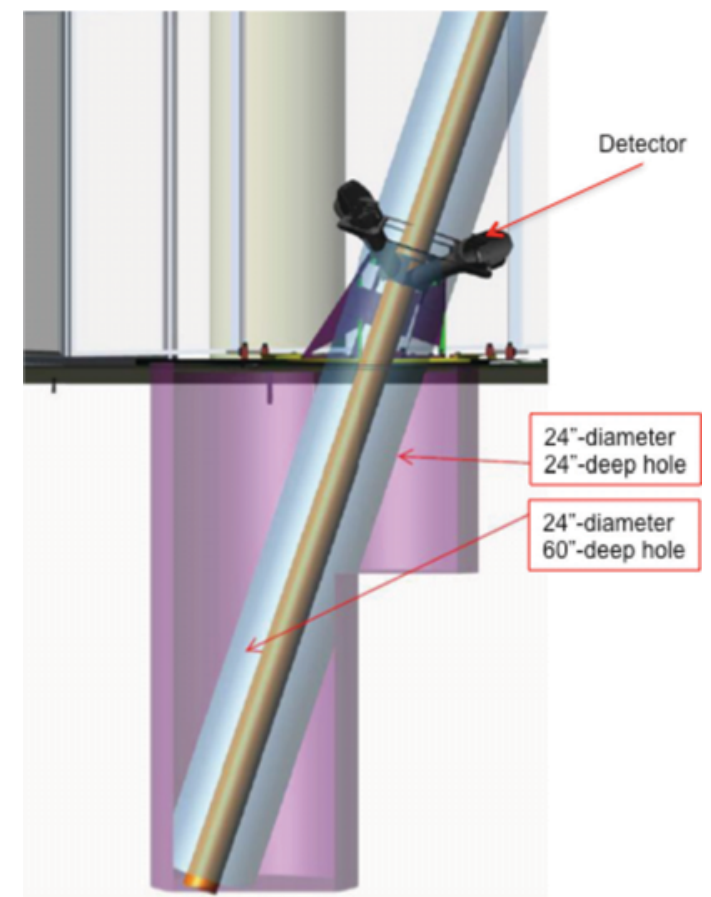

Figure 3. Get lost hole at the -33 '9" floor.

\section{Simulation of radiation backgrounds inside the Target Bay}

Fusion neutrons generated by fuel burn and secondary neutrons and gammas resulting from fusion neutron interactions with structures present inside and outside the TC contribute to the radiation background. Inside the TC, neutrons interact with the Cryogenic Target Positioner, and three DIM instruments. Neutrons also interact with the stainless steel first wall panels, aluminum TC, gunite shield, port covers, and the Final Optics Assemblies. Gammas induced by neutron interactions with different structures inside and outside the TC contribute to the gamma background. A detailed MCNP [4] model has been developed to simulate the expected neutron and gamma backgrounds at different locations inside the NIF facility. The model includes most of the major equipment inserted in the TC and the major diagnostics and structures inside the TB. A 3-D visualization [5] of the detailed TB model is shown in Fig. 4.

The as-built facility drawings were used to create the MCNP model. The TC is made of a $10 \mathrm{~cm}$-thick aluminum shell surrounded by $40 \mathrm{~cm}$ of borated concrete "gunite". The TC includes 48 indirect-drive and 24 direct-drive laser beam ports as well as 120 diagnostics ports. The NIF is currently configured for "indirectdrive", which as mentioned before focuses laser beams onto the interior wall of the hohlraum, thereby generating $\mathrm{x}$-rays that heat the capsule contained within. 
The NIF beampath would need to be reconfigured to operate in "direct-drive" mode, where the laser beams focus directly onto the capsule and a hohlraum is not used. All unused diagnostics and direct-drive ports are unshielded and only covered with $\sim 5 \mathrm{~cm}$-thick port covers made of aluminum alloy. Indirect-drive ports are connected to full models of the Final Optics Assemblies (FOA). The TB shielding walls are made of 1.83 m-thick concrete.

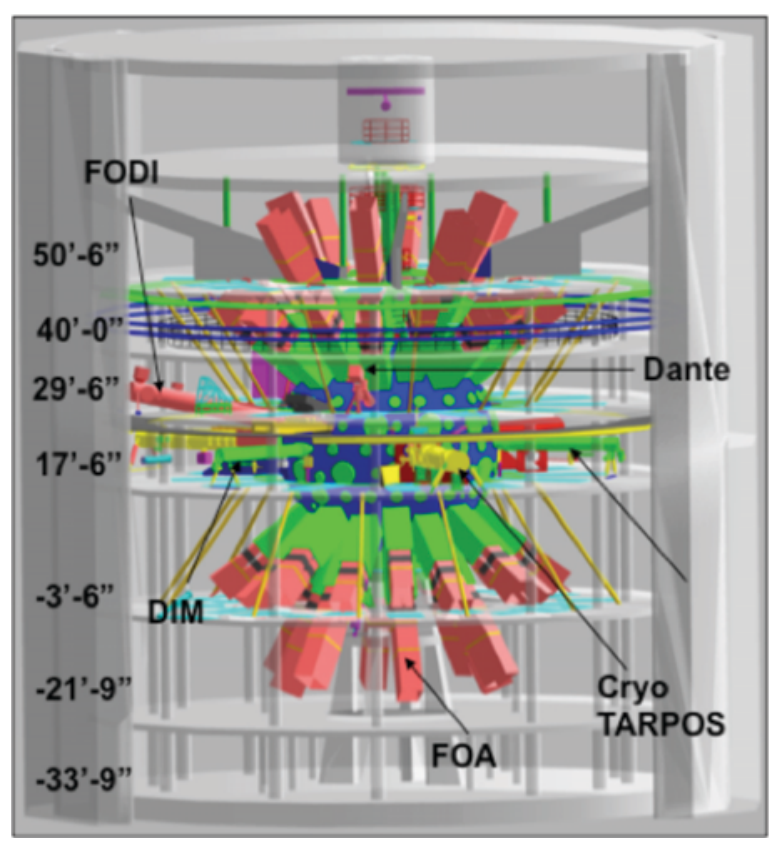

Figure 4. MCNP model of the Target Bay.

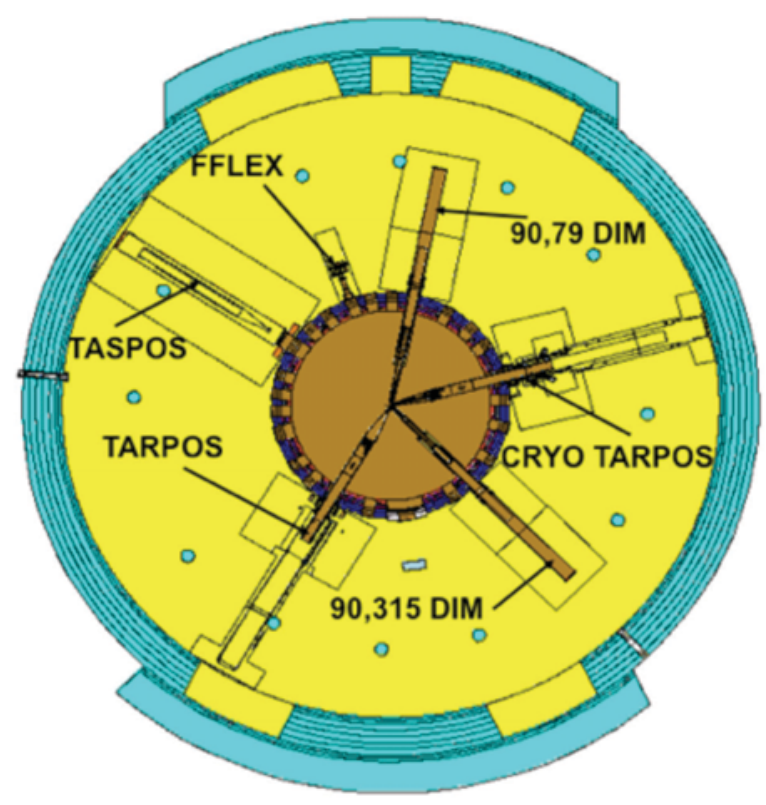

Figure 5. Equatorial view of the Target Bay model.

A two-dimensional view of MCNP model of the TB is shown in Fig. 5. In this view of the TC center, all positioners and DIM instruments are inserted inside the chamber during the shot. Finally, a detailed MCNP model of the nToF detector was separately developed and added to the facility model to be utilized in the background calculations. A 3-D visualization of the detector's MCNP model is shown in Fig. 6.

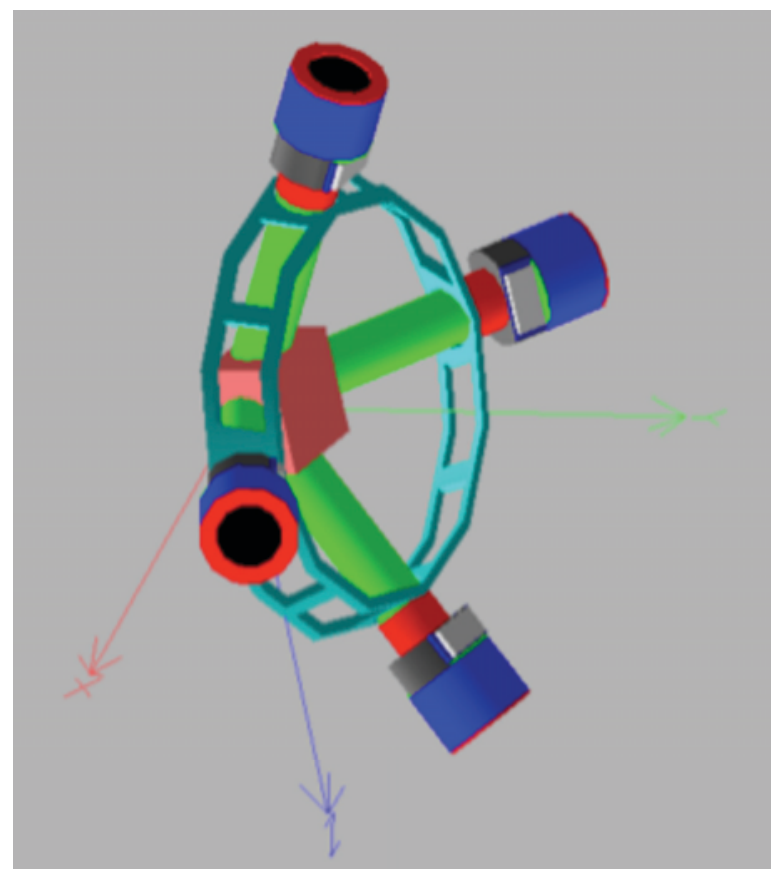

Figure 6. MCNP model of the nToF detector.

The TB model has been utilized in the simulation of expected neutron and gamma fluences throughout the TB [6]. The D-T neutron source is modeled as a volumetric source filling the hohlraum. The neutron cross section data were taken from the FENDL-2.1 cross section database [7]. Photon cross sections are derived from the ENDF/B-VI.8 data library. The neutron and gamma fluence maps are created for each of the TB floors. A Cartesian mesh size of $30 \mathrm{~cm}$ x $30 \mathrm{~cm}$ x $30 \mathrm{~cm}$ is used to create the maps. To reduce the statistical error associated with the simulation, particle splitting and Russian roulette are used throughout the geometry. The typical statistical error associated with the calculated neutron and gamma fluences inside the TB was less than $10 \%$.

The calculated fluences were also used to create a set of dose maps for all floor levels. Silicon kerma factors were used to calculate the dose [8]. Figures 7 and 8 show the simulated neutron and gamma doses (in $\mathrm{mSv}$ to $\mathrm{Si}$ ) for the $-21^{\prime}$ ' " floor level during a $20 \mathrm{MJ}$ shot $\left(7.1 \times 10^{18}\right.$ neutrons), respectively. This floor level contains the final mirrors that are used to focus the laser beams onto the target in the center of the TC. The 192 laser beams enter the TC through 48 indirect-drive beam ports. Following fusion reactions, neutrons and gamma radiation streaming through the lower 24 beam ports passes through the final mirrors with minimal scattering and end up colliding with the -21 '9" concrete floor. The concrete floor is $30.48 \mathrm{~cm}$-thick, and for all practical 
purposes, it acts as a beam dump for neutrons and gamma streaming out of the TC. The lower 24 beam ports form two rings. The 8 hot spots shown on Fig. 7 in red near the TCC represent dose due to neutrons streaming through the inner lower ring of beam ports. Dose due to radiation streaming through the outer lower ring can be seen in the 16 spots near the TB wall.
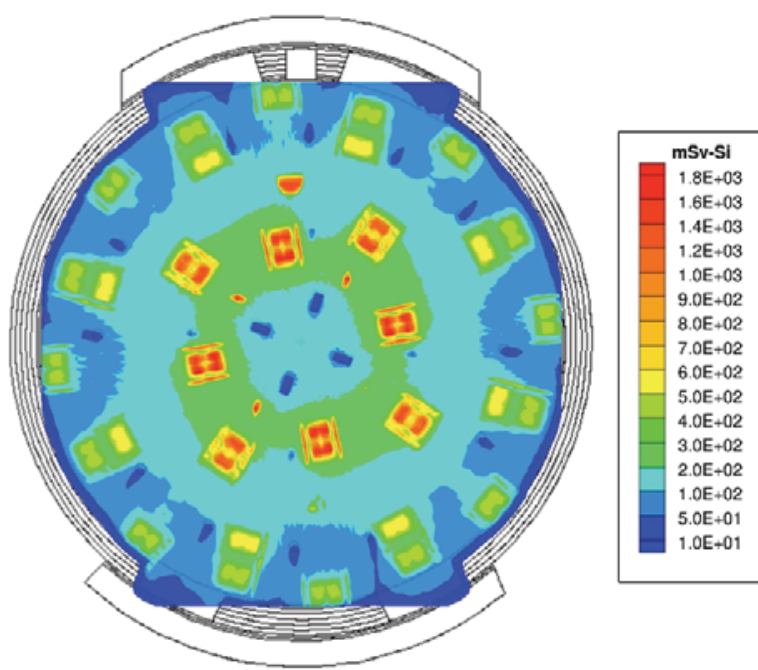

Figure 7. Neutron dose in the -21'9” TB floor.
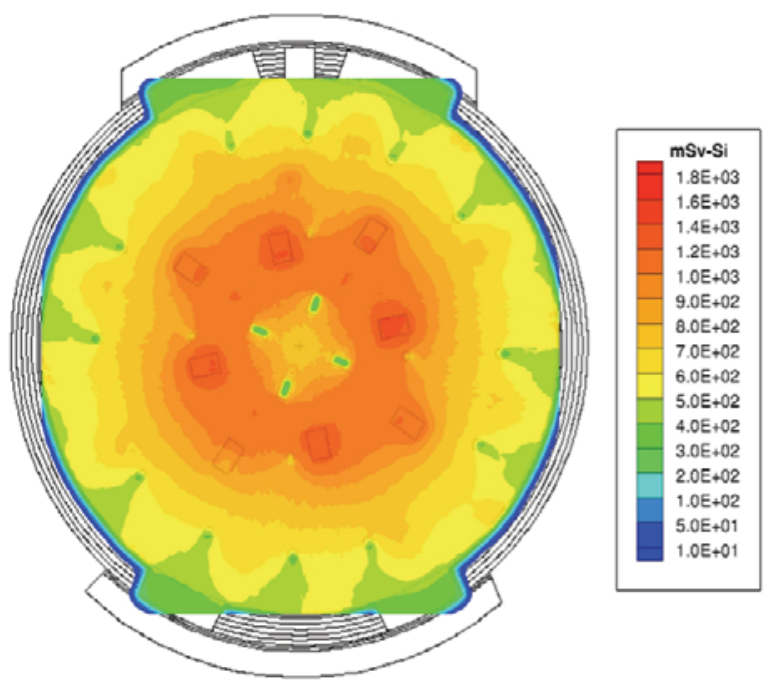

Figure 8. Gamma dose in the $-21^{\prime}{ }^{\prime \prime}$ " TB floor.

Neutrons and gamma passing through the -21'9" concrete floor create hot spots in the -33 ' 9 " floor level, in the vicinity of the SP nToF detector location. Figures 9 and 10 show the expected neutron and gamma doses in the -33'9" floor level, respectively. As can be seen, the location of the detector experiences a moderate neutron dose but a relatively high gamma dose. The gamma radiation is mostly produced by the neutron interactions with the $-21^{\prime} 9$ " concrete floor. The gamma dose is problematic because it arrives at the detector location well before the primary neutron signal. This early arrival would have an impact on the measured signal from the high energy RIF neutrons that are expected to arrive early at the detector location during the same time as the gamma radiation.

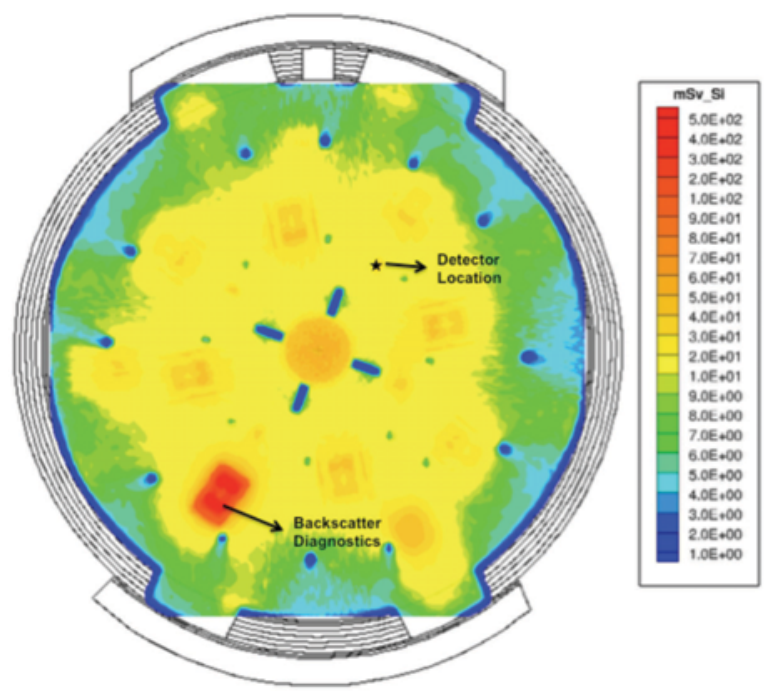

Figure 9. Neutron dose in the -33 ' 9 ” TB floor.

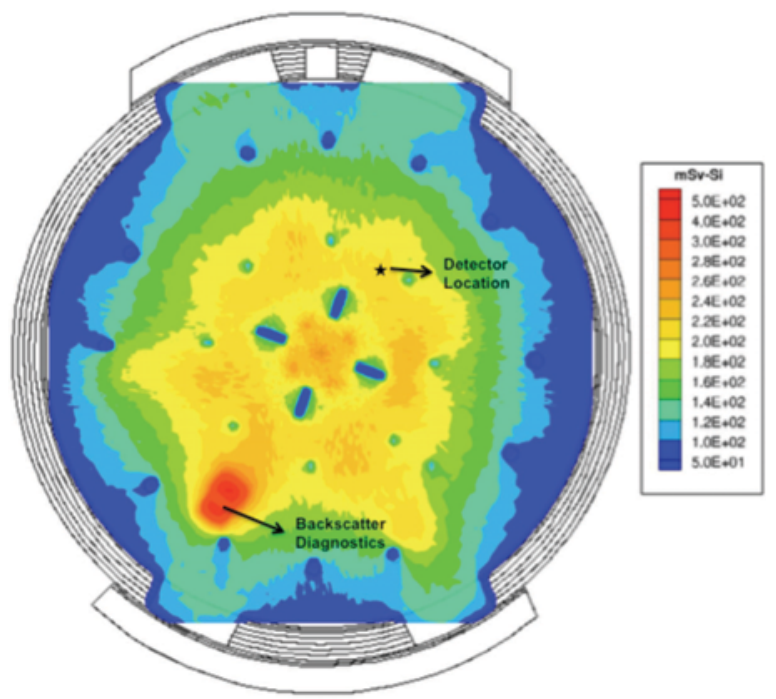

Figure 10. Gamma dose in the $-33^{\prime} 9$ " TB floor.

\section{Reducing detector background}

The SP nToF detector is located at a distance of about $1.4 \mathrm{~m}$ above the $-33^{\prime} 9$ " floor, and the front face of the bibenzyl scintillator is at a distance of $17.88 \mathrm{~m}$ from the TC center [9]. The detector has been installed inside the NIF facility and started taking data during shots with lower neutron yield $\left(10^{15}\right.$ to $\left.10^{16}\right)$. During cryogenic shots, failure to identify the primary neutrons scattering back from the get lost hole could interfere with the measured signal due to the $10-12 \mathrm{MeV}$ DS neutrons (due to the primary $14.1 \mathrm{MeV}$ neutron interactions with the target fuel). To solve this problem, data were taken during shots with non-cryogenic, D-T gas-filled "exploding pusher" targets. During these shots, no DS 
neutrons are expected and hence it is possible to identify and separate the measurement due to primary neutrons passing through the scintillator and scattering back from the get lost hole in the TB floor. On the other hand, early measurements indicated the need to develop a shielding solution to significantly reduce the dose due to gammas arriving at earlier times, before the main $14.1 \mathrm{MeV}$ neutron pulse.

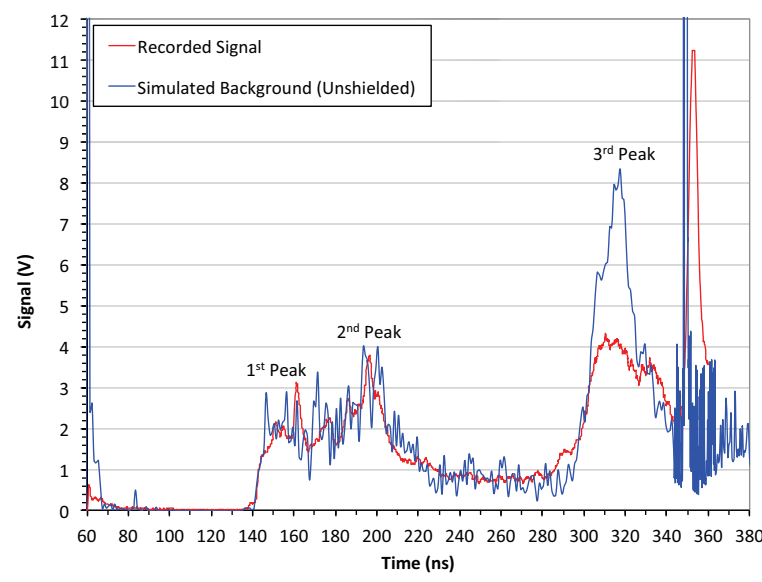

Figure 11. Comparison between simulated background and recorded signal during a low yield shot.

The energy deposited into the SP nToF bibenzyl scintillator has been simulated for a $14.1 \mathrm{MeV}$ neutron source. A comparison between the detector recorded signal and simulated gamma background is shown in Fig. 11. The data were taken during a shot with a yield of $\sim 10^{16}$ neutrons. As shown in the figure, there is a very good agreement between the recorded data and simulated background. The measured data shows a large gamma signal recorded by the scope at $\sim 60 \mathrm{~ns}$ following the shot. The gamma is generated by neutron interactions with masses near the TCC. The hohlraum, target positioner and DIM snouts represent the masses near the TCC. The gamma signal associated with the primary neutron interactions with the scintillator is recorded at $\sim$ $350 \mathrm{~ns}$ following the shot. The measured data shown on the figure were adjusted to match the arrival time simulated by the MCNP simulations. The actual 14.1 $\mathrm{MeV}$ neutron signal is usually recorded by the scope at $370 \mathrm{~ns}$, which is about $20 \mathrm{~ns}$ more than the time it takes the neutrons to reach the front face of the scintillator. The additional time represents the time periods between firing the laser and when it hits the hohlraum in addition to the time to nuclear burn of the D-T fuel.

Both of the recorded signal and simulation results pointed out to a problem with the unexpected arrival of early gammas before the $14.1 \mathrm{MeV}$ neutron signal. The early gammas arrive at the detector between $\sim 140-340$ ns. There are three different gamma peaks, the first peak between $\sim 140-170 \mathrm{~ns}$, the second peak is between 170220 ns with the final and largest peak is between 300 $340 \mathrm{~ns}$. A careful examination of potential sources for the early gamma radiation identified them as a product of fusion neutron collisions with several structures inside the TB. The first peak is due to gammas generated by neutron interactions with the TC first wall steel panels, the TC aluminium wall, and the gunite. The second peak is due to gammas generated by neutron interactions with the TB concrete floor at the -3 ' 6 " floor level. The third and largest peak is due to gammas generated by neutrons colliding with the -21 '9" concrete floor. As mentioned in the previous section and shown in Fig. 7, the concrete floor at the $-21^{\prime} 9$ " floor level acts as a beam dump for neutrons and gammas streaming out of the TC and traveling through the open laser beam ports. Even though the gammas are generated at different times by neutron collisions with different structures, they all stream through the lower rings of the laser beam ports before colliding with the -21 '9" floor. The early gammas arriving before the $14.1 \mathrm{MeV}$ neutron signal travel through the inner lower ring ( 8 beams). On the other hand, neutrons and gammas passing through the outer lower ring (16 beams) travel for longer distances before colliding with the -21'9" floor. They are mostly responsible for much smaller gamma peaks that arrive at the detector after the arrival of the $14.1 \mathrm{MeV}$ neutrons.

The early arrival of the gamma radiation interferes with measurement of the RIF neutrons. The RIF neutrons can have energies up to $\sim 30 \mathrm{MeV}$ and are produced by up-scattered deuteron or triton undergoing $\mathrm{D}-\mathrm{T}$ reaction with thermal ions. The RIF neutrons are very difficult to measure as they represent $\sim$ five orders of magnitude lower than the primary $14.1 \mathrm{MeV}$ neutrons. They are a very important measure of the cold fuel density [10]. It quickly became clear that there is a need for shielding the detector to eliminate the gamma background during the period before the arrival of the 14.1 MeV neutrons at the detector.

A set of MCNP simulations with the full TB model were conducted to facilitate the design of a new shielding scheme with the goal of reducing the early gamma background by about an order of magnitude. The options of using shielding made of tungsten or carbon steel were examined. A picture of the proposed shielding design is shown in Fig. 12. The shielding box fully surrounded the detector except for the $11.43 \mathrm{~cm}$ diameter hole on the top to allow for clear passage of the primary neutron beam.

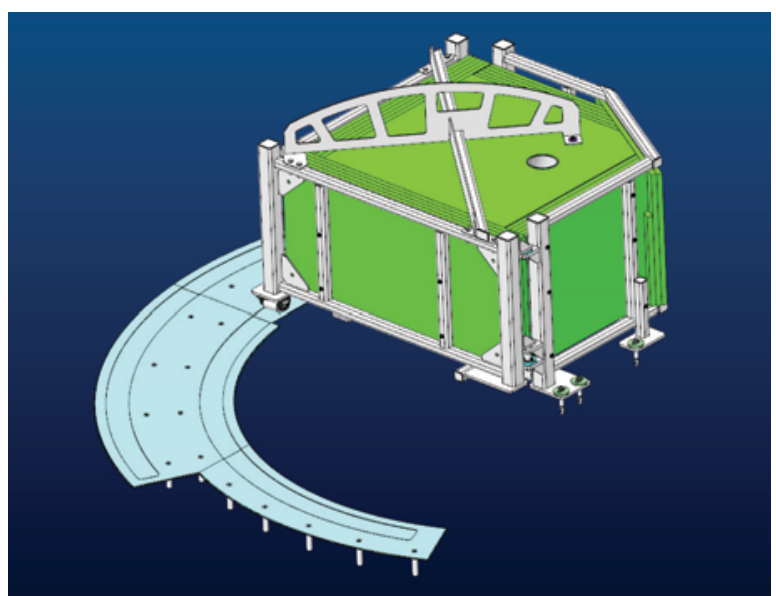

Figure 12. Conceptual design of the nToF detector shielding. 
As shown in Fig. 13, a $5 \mathrm{~cm}$-thick tungsten shield option successfully reduced the energy deposition in the scintillator during the period of concern by about an order of magnitude. Tungsten is an excellent gamma shield but has the known disadvantage of a higher weight and cost than steel. As an alternative to tungsten, a similar reduction in the gamma dose could be achieved through the use of a carbon steel shield.

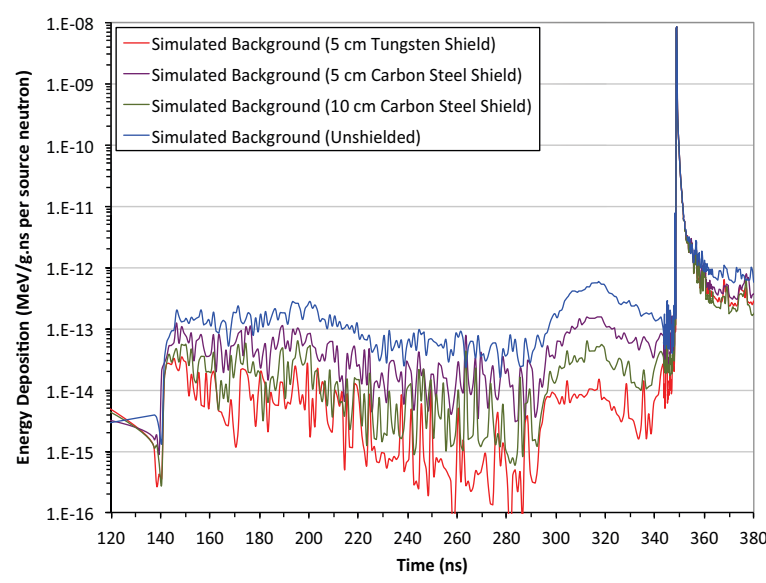

Figure 13. Energy deposition in the nToF scintillator for different shielding options.

As shown in Fig. 14, simulations performed with the proposed shielding options significantly reduced the expected detector signals due to early gamma background. Using a $5 \mathrm{~cm}$-thick tungsten shield would eliminate the signal arriving at the detector as a result of gamma production by neutron collisions with the different structures inside the TB. A somewhat similar reduction in the recorded signal could also be achieved by using a $10 \mathrm{~cm}$-thick carbon steel shield. The proposed shielding solution will allow the detector to successfully record signals associated with the RIF neutrons.

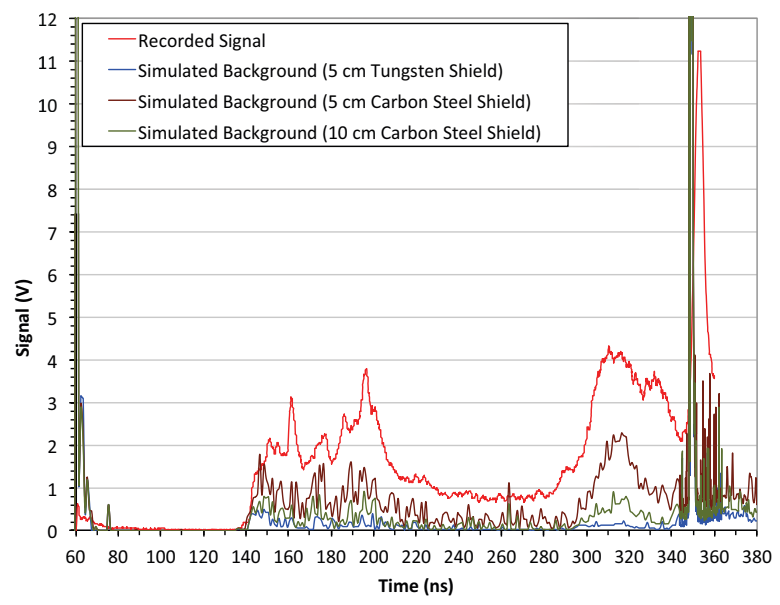

Figure 14. Comparison between simulated background with shielding in place and recorded signal during a low yield shot.

\section{Summary}

A South Pole (SP) neutron time of flight (nToF) detector has been fielded in the National Ignition facility (NIF) to measure neutron yield, ion temperature, and neutron downscattering in the cold fuel for D-T implosions. The SP nToF is located in the lowest floor level of the NIF's Target Bay and at a distance of $\sim 18 \mathrm{~m}$ from the Target Chamber Center. The detector utilizes a solid bibenzyl scintillator and four photomultiplier tubes. Initial measurements indicated the need for installation of additional shielding to reduce the gamma background during a period before the arrival of the primary 14.1 $\mathrm{MeV}$ neutrons to the detector. Gamma arrival during this time period interferes with measurement of the higher energy "reaction-in-flight" (RIF) neutrons. The RIF neutrons can have energies up to $\sim 30 \mathrm{MeV}$ and are produced by up-scattered deuteron or triton undergoing $\mathrm{D}-\mathrm{T}$ reaction with thermal ions. The RIF neutrons are very difficult to measure as they are at about five orders of magnitude lower level than the primary neutrons.

The early gammas arrive at the detector between $\sim 140-340$ ns. They are the product of fusion neutron collisions with the TC walls as well as TB concrete floors at the -3 ' 6 " and the $-21^{\prime} 9$ " levels. Even though the gammas are generated at different times by neutron collisions with different structures, they all stream through the lower rings of the laser beam ports before colliding with the -21 '9" floor. A new shielding that will fully surround the detector has been designed. The gamma background has been reduced by about an order of magnitude during the period of concern through the use of a new $5 \mathrm{~cm}$-thick tungsten or 10 -cm thick carbon steel shield.

This work performed under the auspices of the U.S. Department of Energy by Lawrence Livermore National Laboratory under Contract DE-AC52-07NA27344.

\section{References}

1. V. Glebov et al., Rev. Sci. Instrum. 81, 10D325 (2010)

2. R. Lerche et al., Rev. Sci. Instrum. 81, 10D319 (2010)

3. T. Clancy et al., Proc. SPIE 9211, 92110A (2014)

4. X-5 MONTE CARLO TEAM, Los Alamos National Laboratory, LA-UR-03-1987 (2003)

5. MORITZ, http://www.whiterockscience.com

6. H. Khater and S. Brereton, Prog. in Nuclear Sci. and Tech., 2 (2011)

7. D. Aldama and A. Trkov, International Atomic Energy Agency, INDC(NDS)-467 (2004)

8. M. Singh, Lawrence Livermore National Laboratory, UCRL-52850 (1979)

9. R. Hatarik et al., Rev. Sci. Instrum. 83, 10D911 (2012)

10. A. Hayes et al., Journal of Physics: Conference Series 717, 012022 (2016) 